\title{
A financial audit on hospital costs in an inpatient Psychiatry Unit at Teaching Hospital, Anuradhapura, Sri Lanka
}

\author{
JC Udeshika, A Ellepola
}

\section{Abstract \\ Background}

The Sri Lankan public health care system provides free health care. Psychiatric services in the public sector have expanded over the years to reach the whole country. Currently, the cost of care per patient in a government hospital is not calculated directly.

\section{Aims}

To assess the total in-ward expenditure, expenditures incurred in different cost heads, average per patient cost and cost per patient day in a psychiatry unit at Teaching Hospital, Anuradhapura, Sri Lanka.

\section{Methods}

A financial audit was carried out at Teaching Hospital, Anuradhapura. All patients admitted for in-patient psychiatry care from $1^{\text {st }}$ to $30^{\text {th }}$ of September 2019 were included. Data were collected retrospectively from patient documentations and hospital accounting using a data extraction form.

\section{Results}

Ninety patients, 48 (53.3\%) females and 42 (46.7\%) males, were included in the study, with 711 in-patient days. The average length of stay was 7.9 days. The mean age was 39.8 years, ranging from 16 to 75 years, and most were married (55.6\%). Schizophrenia and depressive disorder were the commonest diagnoses. The total cost per month in the psychiatry unit was LKR 3321 050.60, with an average of LKR 110701.70 per day. The average cost per patient per day was LKR 4 670.96. Staff wages accounted for $56.27 \%$ of the total expenses, and medications and other consumables accounted for $13.01 \%$.

\section{Conclusions}

Clinicians can carry out cost analysis with data available to them. The results can be used to efficiently allocate resources from the total budget in the given institution, and to implement cost reduction plans to use limited resources effectively.

Key words: financial audit, cost analysis, expenditure, psychiatry, in-patient care

SL J Psychiatry 2020; 11(2): 26-30

\section{Introduction}

Teaching Hospital, Anuradhapura has two psychiatric units, i.e., a University and a Health Ministry Unit, catering to all patients in the Anuradhapura district. These units provide in-ward psychiatric care, psychotherapy, electroconvulsive therapy, consultancy services and follow up, psychiatric liaison services, outpatient clinics, day centre services, as well as outreach programmes for community psychiatry services. The Health Ministry unit consists of separate male and female wards, with a total bed strength of 50, and an average of 80-100 admissions per month. Staff of the unit include, dedicated nursing staff, medical officers, two consultant psychiatrists and other assisting staff.
Sri Lanka has a widespread healthcare system with inpatient care, medication, investigations and other health services provided free of charge for patients in the government sector. People in Sri Lanka receiving care in the government sector are often unaware of the expenses the government bears in providing them with medical care. The increasing demands and rising costs in providing health care places a heavy burden on the economy of the country. It would be helpful to know the overall cost, as well as cost per patient, in a given treatment specialty, in order to analyze ways in which to plan expenditure and allocate budgets appropriately, as well as to minimize unwanted expenses. This was demonstrated in a cost review of an acute mental health care unit in a South African general specialist hospital, 
which showed that only $2.4 \%$ of total budget of the hospital was utilized for mental health services, and that in mental health, staff wages was only $2.8 \%$ of the total expenditure (for the year 2007/08) (1). But $4.6 \%$ of services provided in the hospital, in terms of in-patient days, was provided by the psychiatric unit (1). A financial audit would give an objective examination of the importance of psychiatry services. This will also help policy makers gather more reliable quantitative data on the use of allocated funds and help to reduce inequalities of funding and resource allocations among different units.

Several audits on costs of patient care have been carried out in Sri Lanka with regards to non-psychiatry specialties $(2,3)$. To the best of our knowledge, no direct data is available on the cost analysis of psychiatric inpatient care received by patients in this country. The objective of this audit was to assess the total inward expenditure in a psychiatry unit at Teaching Hospital Anuradhapura, Sri Lanka, to analyse expenditures incurred in different cost heads and to estimate per patient cost per month and per day, in the psychiatry unit.

\section{Methods}

This audit was carried out for the period from the $1^{\text {st }}$ to the $30^{\text {th }}$ of September 2019, at the Health Ministry Psychiatry Unit, Teaching Hospital, Anuradhapura. Data on consumables and services rendered for a given patient was analyzed retrospectively from the bedhead tickets. Bedhead tickets were used as a source of information since this comprehensively documents the patients' care during the entire hospital stay. Data were collected using a data extraction form designed for the study and all data entry was done by the same investigator to conform uniformity. Cost estimations were done with the guidance of the World Health Organization (WHO) manual for hospital cost analysis (4). While all hospital costs were calculated, any direct or indirect costs incurred by the individual patient herself/himself were not taken into account.

Cost in human resources including doctors, nursing officers, occupational therapist, social worker and minor staff working only for the psychiatry unit were calculated considering salaries for the year 2019. Costs for security personnel and cleaning staff were calculated based on their monthly wages. Administrative costs were estimated based on the total administrative costs in the hospital, by calculating allocated cost to the staff of the Psychiatry Unit. Capital costs such as for building, furniture and reusable instruments and recurrent expenditure for maintenance, and transport were not accounted for in this study.
Medication were costed for as per the medication price list for the year 2019, as indicated by the Medical Supplies Division (MSD) of the Ministry of Health (5). Consumables such as syringes, gloves, plasters were accounted for based on the monthly purchase order book maintained in the ward, and unit costs were assigned according to the price list of MSD items for 2019. The cost of investigations were established using an average cost from three independent private laboratories, after removing the margin of profit that is usually incorporated in their fee. The margin of profit differed depending on the investigation, ranging from 5\% to $20 \%$.

The monthly electricity cost was calculated with the assistance of engineers from the Ceylon Electricity Board (CEB), who allocated a unit cost considering the total hospital expenditure and the approximate consumption of watt hours of electricity in the unit. Water consumption, telephone usage and waste disposal costs were also estimated for the month considering the total hospital expenses and by calculating a value to the unit proportionate to the admissions in a unit. The amount spent on meals for patients were estimated using the expenditure in the hospital kitchen monthly, and laundry costs by using the monthly hospital expenses for laundry and assigning a value proportionate to admissions in the unit per month and the total inward days. All data were double-entered and cross checked for consistency. Data were analysed using SPSS version 24 statistical software package.

\section{Results}

A total of 90 patients were admitted to the Psychiatry Unit during the period $1^{\text {st }}$ to $30^{\text {th }}$ September 2019, including 48 (53.3\%) females and 42 (46.7\%) males. A cumulative 711 inpatient days were spent in hospital, by these patients. The average length of stay was 7.9 days per patient, with a range from 01 to 29 days. The median number of stayed days was 11 . The age of patients ranged from 16 to 75 years (mean age 39.8 years). Schizophrenia and depressive disorder were the most frequent diagnoses among all patients admitted during the study period. The most common comorbid disorders were hypertension and diabetes mellitus.

The total cost per month in the Psychiatry Unit was LKR 3321 050.60, with an average of LKR 110701.70 per day. The average cost per patient was LKR 4670.96 per day, and LKR 36900.56 per patient over the month (Table 1). Staff wages (including two consultant psychiatrists, a registrar in psychiatry, four medical officers, one occupational therapist, one social worker, sixteen nursing officers and eight minor staff) accounted for $56.27 \%$ of the total expenses during the month and $13.01 \%$ was spent on drugs and other consumables (Figure 1). 
Table 1. Itemized expenses for the Psychiatry Inpatient Unit, during the study period

\begin{tabular}{|c|c|}
\hline Cost head & Cost (LKR) \\
\hline Staff wages & 1868915.00 \\
\hline Psychiatric Drugs & 45961.99 \\
\hline Other drugs & 70330.26 \\
\hline Investigations & 125880.00 \\
\hline Psychotherapy & 41250.00 \\
\hline ECT & 200000.00 \\
\hline Referrals & 15050.00 \\
\hline Other consumables & 315732.37 \\
\hline Administration & 35360.95 \\
\hline Diet & 348560.75 \\
\hline Electricity & 125800.00 \\
\hline Water & 45729.00 \\
\hline Telephone & 2952.80 \\
\hline Laundry & 7872.60 \\
\hline Waste disposal & 7654.88 \\
\hline Cleaning services & 28000.00 \\
\hline Security services & 36000.00 \\
\hline Total & 3321050.60 \\
\hline Average cost per day & 110701.70 \\
\hline Average cost per patient per month & 36900.56 \\
\hline Average cost per patient per day & 4670.96 \\
\hline
\end{tabular}

\section{Expenses in each cost head as a percentage of total expenses}
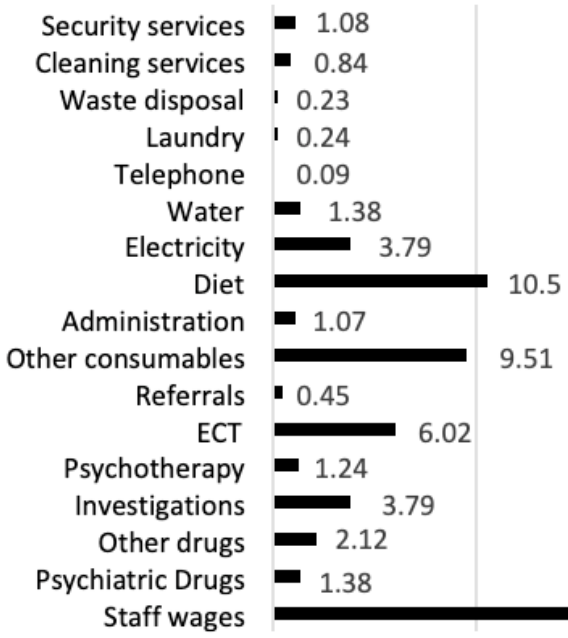

Proportion of cost per head, as a percentage of total cost

Figure 1. Proportion of expenses per head, as a percentage of the total expenses for the Psychiatry Unit, during the study period. 


\section{Discussion}

The findings of this one-month audit indicate the largest proportion of the expenses were spent on wages for staff of the psychiatry unit (56.27\% of total costs), and the second highest monthly expense was on the patients' diet. Similar findings were reported by a South African cost analysis of an acute mental health unit, with staff salaries being $62.8 \%$ of the total expenditure (1). A study done in a surgical unit in Sri Lanka in 2003 found that the highest proportion of the cost (30\%) was for basic hospital charges (2). The results of a study done in 2009 in an Intensive Care Unit (ICU) revealed that the cost per bed, per day was Rs. 9359.07 (for maintenance of the bed if no patients for beds) and Rs. 16712.63 (if patients admitted) - indicating an additional cost of Rs.7353.56 if a patient got admitted (3). Based on our findings, the cost for psychiatry inpatient care (per day) is approximately 4 times less than when compared to the cost of an occupied ICU bed.

Costs of other consumables; electroconvulsive therapy (ECT), electricity and investigations are also significant. To some degree, expenses per patient can be reduced by reducing the duration of inpatient stay. One option would be to consider community based care, but the costs of community care also need to be taken into account. In this audit, costs for medication were less compared to other costs - this may represent more cost effective purchasing of medications by government authorities, but this needs to be compared against patient outcomes. Rational and per protocol use of investigations and other consumables can also help reduce costs.

A more detailed analysis on the breakdown of costs according to diagnosis of the patient may provide data on how much the government spends on patients with different psychiatric illnesses. This may help budget and resource allocations according to the need, lessening the burden of untreated or under-treated psychiatric patients on the society and economy.

Health care needs continue to expand with time, leading to exhaustion of limited resources, and therefore a costbenefit analysis can help utilization of available resources in an optimum way. A financial audit can be easily conducted with the data available in ward and different departments of the hospital. Analysis of data from financial audits can help implement cost saving measures directed at increasing efficiency with a minimum cost. This is particularly important in developing countries such as Sri Lanka, and allocation of a separate mental health budget would allow funding to be allocated to the most needed resources, and would also allow monitoring of how funds are spent. Resource allocations would then be more rational and transparent within an institution and the healthcare system as a whole. In the future, electronic medical records, computing equipment, technical expertise, administrative support and trained, dedicated staff would be useful to have a more comprehensive and accurate cost analysis with a bottom-up approach (1).

\section{Limitations}

On a few instances the patients may have bought medication from the private sector due to unavailability in the government hospital, but we were unable to calculate these costs due to unavailability of pharmaceutical bills, which is a limitation. Furthermore, capital costs such as for building, furniture and reusable instruments and recurrent expenditure for maintenance, and transport were not accounted for in this study. Expenditure for community psychiatry services such as the depot (fluphenazine deconate) program, community psychiatry nursing and out-reach clinics could be much higher than anticipated. In this study, we were only able to gather data on in-patient psychiatry costs. A more comprehensive study, including hidden costs, will reveal a better picture of the health expenses of the government.

\section{Conclusions}

Maintaining an acute psychiatry unit is costly, but it is a paramount need within the healthcare system. The findings of this study shed light on areas that need cost reduction and help to provide objective guidance to administrators when deciding on budget allocations. A comprehensive management plan for each patient could minimize the number of in-patient days and patient admissions, which would potentially reduce the health cost. The largest proportion of expenses is on staff wages, which reflects the fact that human resources are a key part in the management of patients in psychiatry.

This study could be used to provide a framework for future cost analysis in other psychiatry units in the country. More detailed analysis in the future are needed, for instance regarding expenditure based on the diagnosis of the patient and the costs of community psychiatric services, in order to carry out more targeted health care planning and budgeting.

\section{Acknowledgements}

The authors of this study would like to acknowledge the support extended by the staff of ward 5 and 15, Teaching Hospital, Anuradhapura. The assistance given by the Ceylon Electricity Board and Water Board is also greatly appreciated. Clerical staff of Teaching Hospital, Anuradhapura, who provided information about different costs are gratefully acknowledged. We also thank the staff of the three private laboratories in Anuradhapura who helped to calculate costs of investigations. 


\section{Statement of contribution}

Both authors contributed to the conceptualization and writing of the article.

\section{Conflicts of interest}

None declared.

JC Udeshika, Registrar in Psychiatry, Teaching Hospital, Anuradhapura, Sri Lanka

A Ellepola, Consultant Psychiatrist, Teaching Hospital, Anuradhapura, Sri Lanka

Corresponding author: A Ellepola

Email: ellepola@gmail.com

iD http://orcid.org/0000-0001-9699-2777

\section{References}

1. Van Rensburg ABRJ, Jassat W. Acute mental health care according to recent mental health legislation Part II. Activity-based costing. African Journal of Psychiatry 2011; 14(1): 23-29.

2. Malalasekara AP, Ariyaratne MHJ, Fernando R. Cost accounting in a surgical unit in a teaching hospital - A pilot study. CMJ 2003; 48: (3).

3. Dassanayaka JH. Cost per patient day in Intensive Care Unit and cost awareness among Medical Officers and Nursing staff at Base Hospital, Horana Sri Lanka. PGIM e-Repository 2009.

4. Shephard DS, Hodgkins D, Anthony YE. Analysis of hospital costs: a manual for managers, Geneva: World Health Organisation 2000.

5. Medical supplies division: Price list report. Department of Health Services, Colombo. 2019. 